\title{
EMBEDDING GROUPS OF CLASS TWO AND PRIME EXPONENT IN CAPABLE AND NONCAPABLE GROUPS
}

\author{
ARTURO MAGIDIN
}

(Received 19 June 2008)

\begin{abstract}
We show that if $G$ is any $p$-group of class at most two and exponent $p$, then there exist groups $G_{1}$ and $G_{2}$ of class two and exponent $p$ that contain $G$, neither of which can be expressed as a central product, and with $G_{1}$ capable and $G_{2}$ not capable. We provide upper bounds for $\operatorname{rank}\left(G_{i}{ }^{\mathrm{ab}}\right)$ in terms of $\operatorname{rank}\left(G^{\mathrm{ab}}\right)$ in each case.
\end{abstract}

2000 Mathematics subject classification: primary 20D15.

Keywords and phrases: capable, $p$-group, 2-nilpotent.

\section{Introduction}

A group is called capable if it is a central factor group. Capability plays an important role in Hall's scheme of classifying $p$-groups up to isoclinism [5], and has interesting connections to other branches of group theory. The finitely generated capable Abelian groups were classified by Baer [1]. It is not too difficult to determine whether a given finitely generated group of class two is capable or not by finding its epicentre $Z^{*}(G)$, the smallest subgroup of $G$ such that $G / Z^{*}(G)$ is capable. Computing the epicentre in this setting reduces to relatively straightforward computations with finitely generated Abelian groups; see for example [3, Theorems 4 and 7]. However, currently available techniques seem insufficient to give a classification of capable finitely generated groups of class two along the lines of Baer's result for the Abelian case.

A full classification for the $p$-groups of class two and prime exponent seems a modest and possibly attainable goal; some purely numerical necessary conditions [6, Theorem 1] and sufficient ones [8, Theorem 5.26] are known, and a number of results allow us to reduce the problem to a restricted subclass. If we let $G$ be a $p$ group of class at most two and odd prime exponent, it is not hard to show (for example, using [2, Proposition 6.2]) that if $G$ is a nontrivial direct product, then $G$ is capable if and only if each direct factor is either capable or nontrivial cyclic. We can write $G$ as $G=K \times C_{p}^{n}$ where $K$ is a group that satisfies $Z(K)=K^{\prime}$ and $C_{p}$ is cyclic of order $p$,

The author was supported by a grant from the Louisiana Board of Regents Support Fund.

(c) 2009 Australian Mathematical Society 0004-9727/2009 \$16.00 
and so $G$ is capable if and only if $K$ is nontrivial capable, or $K$ is trivial and $n>1$. If $G$ can be decomposed as a nontrivial central product, $G=C D$ with $[D, C]=\{e\}$ and $\{e\} \neq[C, C] \cap[D, D]$ (where $e$ is the identity of the group), then $G$ is not capable [6, Proposition 1]. Ellis proved that if $\left\{x_{1}, \ldots, x_{n}\right\}$ is a transversal for $G / Z(G)$, and the nontrivial commutators $\left[x_{j}, x_{i}\right], 1 \leq i<j \leq n$ form a basis for $[G, G]$, then $G$ is capable [3, Proposition 9]. We are then reduced to considering groups in a restricted class; we give it a name for future reference.

Definition 1.1. We will denote by $\mathcal{R}_{p}$ the class of all $p$-groups $G$ of odd prime exponent $p$ that cannot be decomposed into a nontrivial central product, with $Z(G)=$ $[G, G]$, and such that if $\left\{x_{1}, \ldots, x_{n}\right\}$ is a transversal for $G / Z(G)$, then there is a nontrivial relation among the nontrivial commutators of the form $\left[x_{j}, x_{i}\right]$, $1 \leq i<j \leq n$.

Unfortunately, the situation appears to be far from straightforward once we reach this point. In particular, as the two main results in this paper show, to determine whether such a $G$ is capable we need a 'holistic' examination of $G$ : there are no forbidden-subgroup criteria for the capability or noncapability of $G$. Explicitly, we show that if $G$ is any group of class at most two and odd prime exponent $p$, then $G$ is contained in groups $G_{1}$ and $G_{2}$, both in $\mathcal{R}_{p}$, and with $G_{1}$ capable and $G_{2}$ not capable.

In Section 2 we prove some properties of the 2-nilpotent product with amalgamation, which is our main construction tool. Section 3 contains our main results.

\section{The 2-nilpotent product with amalgamation}

Groups will be written multiplicatively, and we will use $e$ to denote the identity element of $G ; C_{p}$ denotes the cyclic group of order $p$.

Definition 2.1. Let $A$ and $B$ be nilpotent groups of class at most two. The 2nilpotent product of $A$ and $B$ is defined to be the group $F /[[F, F], F]$, where $F=A * B$ is the free product of $A$ and $B$. We denote the 2-nilpotent product of $A$ and $B$ by $A \amalg^{\mathfrak{N}_{2}} B$.

The 2-nilpotent product was introduced by Golovin [4], with a more general definition that applies to any two groups $A$ and $B$. If $A$ and $B$ are nilpotent of class at most two, then their 2-nilpotent product is their coproduct (in the sense of category theory) within the variety of all groups of class at most two, hence our choice of notation. The elements of $A \amalg^{\mathfrak{N}_{2}} B$ can be written uniquely as $\alpha \beta \gamma$, with $\alpha \in A$, $\beta \in B$, and $\gamma \in[B, A]$; multiplication in $A \amalg^{\mathfrak{N}_{2}} B$ is then given by

$$
\left(\alpha_{1} \beta_{1} \gamma_{1}\right)\left(\alpha_{2} \beta_{2} \gamma_{2}\right)=\left(\alpha_{1} \alpha_{2}\right)\left(\beta_{1} \beta_{2}\right)\left(\gamma_{1} \gamma_{2}\left[\beta_{1}, \alpha_{2}\right]\right) .
$$

A theorem of MacHenry [7] shows that $[B, A] \cong B^{\mathrm{ab}} \otimes A^{\mathrm{ab}}$ via $[b, a] \mapsto \bar{b} \otimes \bar{a}$ (where $\bar{x}$ denotes the image of $x$ under the canonical maps $A \rightarrow A^{\mathrm{ab}}$ and $B \rightarrow B^{\mathrm{ab}}$, and $A^{\mathrm{ab}} \otimes B^{\mathrm{ab}}$ is the usual tensor product of Abelian groups). Note that $A \amalg^{\mathfrak{N}_{2}} B$ 
contains isomorphic copies of $A$ and $B$ and is generated by these copies, so if $A$ and $B$ are both of odd exponent $n$, then so is $A \amalg^{\mathfrak{N}_{2}} B$.

Definition 2.2. Let $A$ and $B$ be nilpotent groups of class at most two, let $H \leq[A, A], K \leq[B, B]$, and let $\varphi: H \rightarrow K$ be an isomorphism. The amalgamated coproduct of $A$ and $B$ along $\varphi$ is defined to be the group

$$
A \amalg_{\varphi}^{\mathfrak{N}_{2}} B=\frac{A \amalg^{\mathfrak{N}_{2}} B}{\left\{h \varphi(h)^{-1} \mid h \in H\right\}} .
$$

It is again easy to verify that $A \amalg_{\varphi}^{\mathfrak{N}_{2}} B$ contains isomorphic copies of $A$ and $B$ whose intersection is exactly the image of $H$ identified with the image of $K$ as indicated by $\varphi$.

Recall that a group $G$ is said to be a central product of subgroups $C$ and $D$ if and only if $G=C D$ and $[C, D]=\{e\}$. The direct product is thus a special case of the central product. If in addition we have that $C \cap D=Z(G)$ then we say that $G$ is the full central product of $C$ and $D$. The central product is said to be trivial if $C \subseteq D$ or $D \subseteq C$. We prove that if $A$ and $B$ are both nontrivial, then $A \amalg_{\varphi}^{\mathfrak{N}_{2}} B$ cannot be decomposed as a nontrivial central product.

THEOREM 2.3. Let $A$ and $B$ be nontrivial groups of class at most two and odd prime exponent $p$, let $H \leq[A, A], K \leq[B, B]$, and let $\varphi: H \rightarrow K$ be an isomorphism. If $G=A \amalg_{\varphi}^{\mathfrak{N}_{2}} B$, then $Z(G)=[G, G]$ and $G$ cannot be expressed as a nontrivial central product.

ProOf. Identify $A$ and $B$ with their images in $G$. Let $\left\{a_{1}, \ldots, a_{m}\right\}$ be a transversal for $A /[A, A]$, and $\left\{b_{1}, \ldots, b_{n}\right\}$ a transversal for $B /[B, B]$. Since $A$ is of exponent $p$, every element of $A$ can be written uniquely in the form $a_{1}^{r_{1}} \cdots a_{m}^{r_{m}} a^{\prime}$ with $0 \leq r_{i}<p$ and $a^{\prime} \in[A, A]$, and similarly for every element of $B$. From the construction of the amalgamated coproduct it follows that $\left\{a_{1}, \ldots, a_{m}, b_{1}, \ldots, b_{n}\right\}$ is a transversal for $G /[G, G]$, and that $[G, G] \cong[A, A][B, B] \times[B, A]$. In particular, the commutators $\left[b_{j}, a_{i}\right], 1 \leq i \leq m, 1 \leq j \leq n$ form a linearly independent subset of $[G, G]$ (viewing the latter as an $\mathbb{F}_{p}$-vector space).

Let $g \in G$; then we may write $g=a_{1}^{r_{1}} \cdots a_{m}^{r_{m}} b_{1}^{s_{1}} \cdots b_{n}^{s_{n}} g^{\prime}$, with $0 \leq r_{i}, s_{j}<p$ and $g^{\prime} \in[G, G]$. We first assert that $g \in Z(G)$ if and only if $r_{i}=s_{j}=0$ for all $i$ and $j$. Indeed,

$$
\left[g, a_{i}\right]=\prod_{j=1}^{m}\left[a_{j}, a_{i}\right]^{r_{j}} \cdot \prod_{j=1}^{m}\left[b_{j}, a_{i}\right]^{s_{j}} .
$$

For this to be trivial, we must have $s_{j}=0$ for each $j$. Symmetrically, computing $\left[g, b_{j}\right]$ we obtain that $r_{i}=0$ for each $i$. Thus, $Z(G)=[G, G]$, as claimed.

Now suppose that $G$ is decomposed as a central product, $G=C D$. We can express $a_{1}=c_{1} d_{1}$ for some $c_{1} \in C, d_{1} \in D$. Let

$$
\begin{aligned}
& c_{1}=a_{1}^{r_{1}} \cdots a_{m}^{r_{m}} b_{1}^{s_{1}} \cdots b_{m}^{s_{m}} c^{\prime}, \\
& d_{1}=a_{1}^{\rho_{1}} \cdots a_{m}^{\rho_{m}} b_{1}^{\sigma_{1}} \cdots b_{m}^{\sigma_{m}} d^{\prime},
\end{aligned}
$$


with $c^{\prime}, d^{\prime} \in[G, G]$; since $c_{1} d_{1}=a_{1}$, we have $r_{1}+\rho_{1} \equiv 1(\bmod p)$, and $r_{i}+\rho_{i} \equiv$ $s_{j}+\sigma_{j} \equiv 0(\bmod p)$ for $2 \leq i \leq m, 1 \leq j \leq n$. Since $\left[d_{1}, c_{1}\right]=e$, and

$$
\left[d_{1}, c_{1}\right]=a^{\prime \prime} b^{\prime \prime} \prod_{i=1}^{m} \prod_{j=1}^{n}\left[b_{j}, a_{i}\right]^{\sigma_{j} r_{i}-s_{j} \rho_{i}}, \quad a^{\prime \prime} \in[A, A], \quad d^{\prime \prime} \in[B, B],
$$

we have $0 \equiv \sigma_{j} r_{1}-s_{j} \rho_{1} \equiv \sigma_{j} r_{1}+\sigma_{j}\left(1-r_{1}\right) \equiv \sigma_{j} \equiv-s_{j}(\bmod p)$. That is, $c_{1}, d_{1} \in A[G, G]$. Since $a_{1} \notin Z(G)$, either $c_{1} \notin Z(G)$ or $d_{1} \notin Z(G)$. If both hold, then we have $C \subseteq C_{G}\left(d_{1}\right) \subseteq A[G, G]$ and $D \subseteq C_{G}\left(c_{1}\right) \subseteq A[G, G]$, so we conclude that $G=C D \subseteq A[G, G]$, which is impossible. Thus, exactly one of $c_{1}$ and $d_{1}$ is noncentral. Without loss of generality say $c_{1} \notin Z(G)$ and $d_{1} \in Z(G)$, so $a_{1}=c_{1} d_{1} \in$ $C Z(G)$.

Since none of $b_{1}, \ldots, b_{n}$ commute with $a_{1}$, and $a_{1} \in C Z(G)$, we must have $b_{1}, \ldots, b_{n} \in C Z(G)$ as well; that is, $B \subseteq C[G, G]$. And since $b_{1} \in C[G, G]$ and none of $a_{1}, \ldots, a_{m}$ commute with $b_{1}$, we must also have $a_{1}, \ldots, a_{m} \in C[G, G]$. Thus, $G=C[G, G]$, and so $[G, G]=[C, C]$ and $D \subseteq C$. Hence the central product decomposition $G=C D$ is trivial, as claimed.

We finish this section by describing the epicentre of an amalgamated coproduct.

TheOREM 2.4. Let $A$ and $B$ be nontrivial groups of class at most two and odd prime exponent $p$, let $H \leq[A, A], K \leq[B, B]$, and let $\varphi: H \rightarrow K$ be an isomorphism. If $G=A \amalg_{\varphi}^{\mathfrak{N}_{2}} B$, then $Z^{*}(G)=\left\{h \in H \mid h \in Z^{*}(A)\right.$ and $\left.\varphi(h) \in Z^{*}(B)\right\}$; that is, if we identify $A$ and $B$ with their images in $G$, then $Z^{*}(G)=Z^{*}(A) \cap Z^{*}(B)$.

Proof. Let $a_{1}, \ldots, a_{m}$ be a transversal for $A /[A, A]$, and $b_{1}, \ldots, b_{n}$ a transversal for $B /[B, B]$. Following Ellis, for $x, y, z \in\left\{a_{i}, b_{j} \mid 1 \leq i \leq m, 1 \leq j \leq n\right\}$ let

$$
J(x, y, z)=[x, y] \otimes \bar{z}+[y, z] \otimes \bar{x}+[z, x] \otimes \bar{y}
$$

and let $S$ be the subgroup of $[G, G] \otimes G^{\text {ab }}$ generated by all such $J(x, y, z)$. By [3, Theorem 7], an element $g \in Z(G)=[G, G]$ lies in $Z^{*}(G)$ if and only if $g \otimes w \in S$ for all $w \in\left\{a_{i}, b_{j}\right\}$. Since $[G, G] \cong[A, A][B, B] \times\left(B^{\mathrm{ab}} \otimes A^{\mathrm{ab}}\right), G^{\mathrm{ab}} \cong A^{\mathrm{ab}} \times B^{\mathrm{ab}}$, and all factors are elementary Abelian $p$-groups, we have that $[G, G] \otimes G^{\text {ab }}$ is isomorphic to

$$
\left(C \otimes\left(A^{\mathrm{ab}} \times B^{\mathrm{ab}}\right)\right) \oplus\left(\bigoplus_{i, k=1}^{m} \bigoplus_{j=1}^{n}\left\langle\left[b_{j}, a_{i}\right] \otimes \overline{a_{k}}\right\rangle\right) \oplus\left(\bigoplus_{j, k=1}^{n} \bigoplus_{i=1}^{m}\left\langle\left[b_{j}, a_{i}\right] \otimes \overline{b_{k}}\right\rangle\right),
$$

where $C$ is the central product of $[A, A]$ and $[B, B]$ obtained by identifying $H$ with $K$ along $\varphi$. An easy computation shows that none of the elements $J(x, y, z)$ has a nontrivial $\left[b_{j}, a_{i}\right] \otimes \overline{b_{j}}$ component. Thus, if $g \in[G, G]$ has a nontrivial $[B, A]$ component, say $\left[b_{j}, a_{i}\right]$, then it follows that $g \otimes \overline{b_{j}}$ does not lie in $S$, so $g$ is not in $Z^{*}(G)$. Thus, $Z^{*}(G) \subseteq[A, A][B, B]$. 
Consider the elements $J(x, y, z)$ in which at least one of $x, y$, or $z$ is equal to $b_{1}$. Unless the other two are in $B$, the generators include nontrivial $[B, A] \otimes\left(B^{\mathrm{ab}} \times A^{\mathrm{ab}}\right)$ components that do not occur in any other generator, and occur in pairs. It is straightforward then that if $g \in[G, G]$ lies in $Z^{*}(G)$, since $g \otimes \overline{b_{1}}$ must lie in $S$ we have that $g$ can be expressed in terms of commutators of $b_{1}, \ldots, b_{m}$; that is, $g \in[B, B]$. By a symmetric argument considering $a_{1}$ instead, we obtain that if $g \in Z^{*}(G)$ then $g \in[A, A]$. Thus, $Z^{*}(G)$ is contained in $[A, A] \cap[B, B]$; recall that this intersection is equal to the identified subgroups $H=K$.

If $g \in H$ lies in $Z^{*}(G)$, then $g \otimes \overline{a_{i}} \in S$ for each $i$, and this readily yields that for all $a \in A, g \otimes \bar{a}$ lies in the subgroup of $[A, A] \otimes A^{\mathrm{ab}}$ generated by all $J\left(a_{i}, a_{j}, a_{k}\right)$; thus $g \in Z^{*}(A)$; symmetrically, since $g \otimes \overline{b_{j}} \in S$ we obtain that $g$ (considered now as an element of $K$ ) lies in $Z^{*}(B)$, so $Z^{*}(G) \subseteq Z^{*}(A) \cap Z^{*}(B)$. Conversely, if $h \in H \cap Z^{*}(A)$ is such that $\varphi(h) \in Z^{*}(B)$, then $h \otimes \overline{a_{i}} \in\left\langle J\left(a_{r}, a_{s}, a_{t}\right)\right\rangle$ for all $i$ and $\varphi(h) \otimes \overline{b_{j}} \in\left\langle J\left(b_{r}, b_{s}, b_{t}\right)\right\rangle$ for all $j$, hence $h=\varphi(h) \in Z^{*}(G)$, giving the desired equality.

\section{Main results}

We now give the promised results.

THEOREM 3.1. Let $G$ be any nontrivial group of class at most two and odd prime exponent $p$. Then there exists a capable group $G_{1} \in \mathcal{R}_{p}$ that contains $G$. If $G$ is nonabelian and capable, then we may choose $G_{1}$ so that $\operatorname{rank}\left(G_{1}^{\mathrm{ab}}\right) \leq \operatorname{rank}\left(G^{\mathrm{ab}}\right)+2$. Otherwise, we may choose $G_{1} \operatorname{such}$ that $\operatorname{rank}\left(G_{1}^{\mathrm{ab}}\right) \leq \operatorname{rank}\left(G^{\mathrm{ab}}\right)+3$.

Proof. We construct $G_{1}$ in two steps. If $G$ is nonabelian and capable, set $G_{0}=G$; otherwise, let $G_{0}=G \amalg^{\mathfrak{N}_{2}} C_{p}$. To obtain $G_{1}$, let $H=C_{p} \amalg^{\mathfrak{N}_{2}} C_{p}$, and let $\varphi$ be an isomorphism between $[H, H]$ and a nontrivial cyclic subgroup of $\left[G_{0}, G_{0}\right]$. Finally, let $G_{1}=G_{0} \amalg_{\varphi}^{\mathfrak{N}_{2}} H$. Since $G_{0}$ is capable, $G_{1}$ is capable; by Theorem $2.3 G_{1}$ is not a nontrivial central product. The identification of the generator of $[H, H]$ with a nontrivial element of $\left[G_{0}, G_{0}\right]$ guarantees the existence of a nontrivial relation among nontrivial commutators of any transversal, hence $G_{1} \in \mathcal{R}_{p}$, as desired. The rank inequality is immediate.

THEOREM 3.2. Let $G$ be any nontrivial group of class at most two and exponent $p$. Then there exists a noncapable group $G_{2} \in \mathcal{R}_{p}$ that contains $G$. If $G$ is nonabelian, then we may choose $G_{2}$ such that $\operatorname{rank}\left(G_{2}^{\mathrm{ab}}\right) \leq \operatorname{rank}\left(G^{\mathrm{ab}}\right)+6$. If $G$ is Abelian, then we may choose $G_{2}$ with $\operatorname{rank}\left(G_{2}^{\mathrm{ab}}\right) \leq \operatorname{rank}\left(G^{\mathrm{ab}}\right)+7$.

Proof. If $G$ is nonabelian, let $H_{1}=C_{p} \amalg^{\mathfrak{N}_{2}} C_{p}$, let $g \in[G, G]$ be nontrivial, and let $H$ be the central product of $G$ and $H_{1}$ identifying $g$ with a generator of $\left[H_{1}, H_{1}\right]$. Since this is a nontrivial central product with $[G, G] \cap\left[H_{1}, H_{1}\right] \neq\{e\}$, it is not capable and $g \in Z^{*}(H)$ by [6, Proposition 1]. Now let $G_{2}=H \amalg_{\varphi}^{\mathfrak{N}_{2}} E$, where $E$ is an extraspecial group of order $p^{5}$ and exponent $p$, and $\varphi$ identifies $g$ with a generator 
of $[E, E]$ (which is in $Z^{*}(E)$ ); since this is an amalgamated coproduct that identifies elements of the epicentres, Theorems 2.3 and 2.4 yield that $G_{2}$ is not capable and lies in $\mathcal{R}_{p}$ (the theorem of Ellis mentioned in the introduction guarantees the existence of a nontrivial relation among nontrivial commutators of any transversal).

If $G$ is Abelian, then let $H$ be the central product of $G \amalg^{\mathfrak{N}_{2}} C_{p}$ with $C_{p} \amalg^{\mathfrak{N}_{2}} C_{p}$ identifying a generator of $C_{p} \amalg^{\mathfrak{N}_{2}} C_{p}$ with a nontrivial commutator in $G \amalg^{\mathfrak{N}_{2}} C_{p}$; this is a noncapable group. We now let $G_{2}=H \amalg_{\varphi}^{\mathfrak{N}_{2}} E$ where $E$ is again the extraspecial group of order $p^{5}$ and exponent $p$, and $\varphi$ identifies elements of the epicentres. Again, $G_{2}$ is not capable and lies in $\mathcal{R}_{p}$. The rank inequalities are immediate.

\section{References}

[1] R. Baer, 'Groups with preassigned central and central quotient group', Trans. Amer. Math. Soc. 44 (1938), 387-412.

[2] F. R. Beyl, U. Felgner and P. Schmid, 'On groups occurring as central factor groups', J. Algebra 61 (1979), 161-177.

[3] G. Ellis, 'On the capability of groups', Proc. Edinburgh Math. Soc. 41(2) (1998), 487-495.

[4] O. N. Golovin, 'Metabelian products of groups', Amer. Math. Soc. Transl. Ser. 2(2) (1956), 117-131.

[5] P. Hall, 'The classification of prime-power groups', J. Reine Angew. Math. 182 (1940), 130-141.

[6] H. Heineken and D. Nikolova, 'Class two nilpotent capable groups', Bull. Aust. Math. Soc. 54 (1996), 347-352.

[7] T. MacHenry, 'The tensor product and the 2nd nilpotent product of groups', Math. Z. 73 (1960), 134-145.

[8] A. Magidin, On the capability of finite groups of class two and prime exponent, reprint=arXiv:0708.2391 (math.GR).

ARTURO MAGIDIN, Mathematics Dept., University of Louisiana-Lafayette, 217 Maxim Doucet Hall, PO Box 41010, Lafayette LA 70504-1010, USA e-mail: magidin@member.ams.org 